Article

\title{
A Multiconductor Model of Power Line Communication in Medium-Voltage Lines
}

\author{
Lesek Franek * and Petr Fiedler \\ Faculty of Electrical Engineering and Communication, Brno University of Technology, Technicka 3082/12, \\ 61600 Brno, Czech Republic; fiedlerp@feec.vutbr.cz \\ * Correspondence: lesek.franek@phd.feec.vutbr.cz \\ Academic Editor: Neville R. Watson \\ Received: 26 May 2017; Accepted: 12 June 2017; Published: 15 June 2017
}

\begin{abstract}
Most power line communication (PLC) models are designed to date simulate power lines as two-wire lines. However, in alternating current (AC) electrical distribution, the two-wire option is seldom applied, and medium-voltage lines are most often based on the three-phase configuration. In this context, the influence of the ground, which constitutes another conductor with specific parameters, cannot be neglected. Two-wire models are characterized by limited accuracy, not allowing us to simulate certain major phenomena affecting PLC. This, for example, could embody the answer to the question of whether it is more advantageous to transmit a signal independently through each phase, reference the signal with respect to another phase or to use the ground as a reference. This paper discusses a multi-conductor model that eliminates the disadvantages outlined above; the proposed model exploits the multi-conductor telegrapher's equations. In order to be able to include medium-voltage (MV)/ low-voltage (LV) transformers in medium-voltage network models, we constituted a transformer model. The designed models were validated on a real medium-voltage network. To be able to evaluate the suitability of the PLC, the noise in the medium voltage network was measured in order to determine the signal-to-noise ratio (SNR).
\end{abstract}

Keywords: power line communication; smart grids, internet of things; machine-to-machine communication

\section{Introduction}

Power line communication (PLC) has been used for almost a hundred years for various purposes within a broad range of fields, including telephony [1], street lighting control, and intercoms [2]. At present, PLC finds wide application in smart grids. PLC technologies could be stratified into three categories [3].

The first category, the ultra-narrowband type of communication, is very slow and present mostly within legacy systems [4]. Ultra-narrowband PLC in smart grids is used mainly in the US in configurations with only few electricity meters behind the transformer, as this type of PLC is able to cross medium-voltage (MV) / low-voltage (LV) transformers and reach high voltage transformers [5-7]. Thus, the PLC network extends over all LV network segments that are connected to the given MV voltage network.

The second class contains narrowband communication, the most common solution for smart grids. This group includes a number of standards and proprietary approaches, which can be subdivided into communication with one carrier frequency and multi-carrier frequencies [4,7]. The range of available frequencies in different parts of the world is assigned by relevant regulatory bodies [8]. One of the most popular standards in this category is PRIME [9]. 
The third type, broadband communication, exhibits a high speed and limited communication range, making it a tool most often employed to bridge the Ethernet connection within a single building. However, applications for smart grids are available too $[4,10]$.

Smart grids embody the introduction of information and communications technologies (ICT) into the distribution network to facilitate cost reduction, increase the power supply quality, and reduce the negative aspects related to the impact of energy production and distribution on the environment. Within the different types of communication used in smart grids, PLC very frequently ensures the bidirectional data exchange between the electricity meters and data concentrators located at substations [11]; from the substations, the data are usually sent to servers via mobile networks.

This paper is intended to introduce a model of PLC over a three-phase medium-voltage alternating current (AC) grid. Moreover, taking advantage of the developed model, the authors attempt to resolve the question of whether it could be possible to communicate between the transformers in a medium-voltage grid, and, if so, what parameters can be expected.

\section{Multi-Conductor Power Line Model}

The proposed multi-conductor power line model is based on the telegrapher's equations for multiconductor lines; more detailed information on the general solution of the differential equations for such lines can be found in, for example, [12,13]. Kirchhoff's laws are used to derive the formulas expressing the voltage and current in the phasor form for the elementary section of the line, which is shown in Figure 1:

$$
\begin{aligned}
& \frac{\partial \boldsymbol{V}(z)}{\partial z}=-(\boldsymbol{R}+j \omega \boldsymbol{L}) \boldsymbol{I}(z), \\
& \frac{\partial \boldsymbol{I}(z)}{\partial z}=-(\boldsymbol{G}+j \omega \boldsymbol{C}) \boldsymbol{V}(z),
\end{aligned}
$$

where $z$ denotes the position at the conductor. One of the conductors is identified as the reference conductor. The matrix $V$ represents the voltage between the individual conductors' nodes and the reference conductor's node, found at the $z$ position of a reference conductor, and the matrix $I$ defines the currents flowing through each of the conductors. Both of these matrices exhibit the size $(n ; 1)$. The matrices $\boldsymbol{R}, \boldsymbol{L}, \boldsymbol{G}$ and $\boldsymbol{C}$ have the dimension $(n ; n)$, where $n$ is the number of conductors without the reference conductor, and they are symmetrical with respect to the diagonal. The resistance, inductance, conductance, and capacitance are specified as per-unit-length parameters.

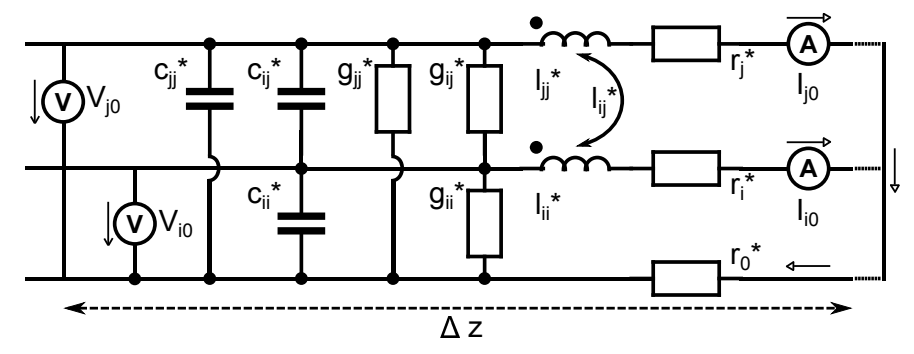

Figure 1. Elementary section of the line.

The matrix $R$ can be expressed as:

$$
\boldsymbol{R}=\left[\begin{array}{cc}
r_{i}+r_{0} & r_{0} \\
r_{0} & r_{j}+r_{0}
\end{array}\right]
$$

The sum of the resistances of the appropriate conductor and reference conductor is found on the main diagonal, whereas the resistance of the reference conductor is then off-diagonal. 
The matrix $L$ can be written as:

$$
\mathbf{L}=\left[\begin{array}{ll}
l_{i i} & l_{i j} \\
l_{i j} & l_{j j}
\end{array}\right]
$$

where the inductances on the diagonal represent the inductance between the relevant conductor and the reference conductor; the mutual inductance of the two relevant conductors is off-diagonal.

The matrix $G$ can be expressed as:

$$
\boldsymbol{G}=\left[\begin{array}{cc}
g_{i i}+g_{i j} & -g_{i j} \\
-g_{i j} & g_{j j}+g_{i j}
\end{array}\right]
$$

where the sum of the conductivity between the relevant conductor and all the other conductors, including the reference conductor, lies on the main diagonal. Outside the main diagonal of the conductivity matrix, the conductivity between the two relevant conductors is marked with the minus sign.

The matrix $C$ can be expressed as:

$$
\boldsymbol{C}=\left[\begin{array}{cc}
c_{i i}+c_{i j} & -c_{i j} \\
-c_{i j} & c_{j j}+c_{i j}
\end{array}\right]
$$

where the main diagonal contains the sum of the capacitances between the appropriate conductor and all of the other conductors, including the reference one. Outside the main diagonal, the capacitance between the two relevant conductors is marked with the minus sign.

To simplify the notation, the impedance and admittance matrices of the transmission lines are defined as follows:

$$
\begin{aligned}
& \boldsymbol{Z}=(\boldsymbol{R}+j \omega \boldsymbol{L}), \\
& \boldsymbol{Y}=(\boldsymbol{G}+j \omega \boldsymbol{C}) .
\end{aligned}
$$

For the second order derivative of voltage according to the line position, we have:

$$
\frac{\partial^{2} V(z)}{\partial z^{2}}=Z Y V(z)
$$

This is a system of $n$ differential equations with $n$ unknowns; in order to solve it, we have to introduce the substitution:

$$
Z Y=T\left[\begin{array}{cc}
\lambda_{1} & 0 \\
0 & \lambda_{2}
\end{array}\right] T^{-1}=T \Lambda T^{-1}
$$

where $T$ is the eigenvector of the matrix $Z Y$, and $\Lambda$ denotes the matrix that has the eigenvalues of the matrix $Z Y$ on its diagonal. By introducing another substitution, where:

$$
V_{m}=T^{-1} V
$$

It is then possible to rewrite Equation (7) as:

$$
\frac{\partial^{2} \boldsymbol{V}(z)}{\partial z^{2}}=\Lambda V_{m}(z) .
$$

Now, we have obtained $n$ independent differential equations with one variable.

By introducing the matrix $\Gamma$ :

$$
\Gamma=\sqrt{\Lambda}
$$


the general solution to these equations can be found in the form:

$$
\boldsymbol{V}_{m}(z)=e^{-\boldsymbol{\Gamma} z} \boldsymbol{V}_{m}^{+}+e^{\boldsymbol{\Gamma} z} \boldsymbol{V}_{m}^{-} .
$$

Note that the exponent functions in Equation (12) are matrix exponents; an exponential of a matrix represents an infinite Taylor series and can be computed taking advantage of the expm() function in computer programs such as Matlab.

The characteristic impedance of the line then equals:

$$
Z_{0}=T \Gamma^{-1} T^{-1} Z \text {. }
$$

The dependence of the voltage and current at the beginning of the line on the voltage and current at its end can thus be expressed as:

$$
\begin{aligned}
\boldsymbol{V}_{\text {in }} & =\boldsymbol{T} \cosh (\boldsymbol{\Gamma} l) \boldsymbol{T}^{-1} \boldsymbol{V}_{\text {out }}+\boldsymbol{T} \sinh (\boldsymbol{\Gamma} l) \boldsymbol{T}^{-1} \boldsymbol{Z}_{0} \boldsymbol{I}_{\text {out }}, \\
\boldsymbol{I}_{\text {in }} & =\boldsymbol{Z}_{0}^{-1} \boldsymbol{T} \sinh (\boldsymbol{\Gamma} l) \boldsymbol{T}^{-1} \boldsymbol{V}_{\text {out }}+\boldsymbol{Z}^{-1} \boldsymbol{T} \cosh (\boldsymbol{\Gamma} l) \boldsymbol{T}^{-1} \boldsymbol{Z} \boldsymbol{I}_{\text {out }} .
\end{aligned}
$$

Note that the hyperbolic functions in Equation (14) are matrix hyperbolic functions; in computer programs, this can be computed using $\operatorname{sinhm}()$ and $\operatorname{coshm}()$ functions.

\subsection{A Line Model for Multiwire Transmission Lines}

Any network element can be described as a multi-port via the matrix $\boldsymbol{M}_{i}$ :

$$
\left[\begin{array}{c}
\boldsymbol{V}_{\text {in }} \\
\boldsymbol{I}_{\text {in }}
\end{array}\right]=\left[\begin{array}{cc}
A_{i} & \boldsymbol{B}_{i} \\
\boldsymbol{C}_{i} & \boldsymbol{D}_{i}
\end{array}\right]\left[\begin{array}{c}
\boldsymbol{V}_{\text {out }} \\
\boldsymbol{I}_{\text {out }}
\end{array}\right]=\boldsymbol{M}_{i}\left[\begin{array}{c}
\boldsymbol{V}_{\text {out }} \\
\boldsymbol{I}_{\text {out }}
\end{array}\right] .
$$

To represent a multi-conductor line as a multi-port matrix, we can use Equation (14), which is already available in the required form.

The load model can be expressed as an admittance matrix, whose inversion will produce an impedance matrix. The admittance matrix can be written as:

$$
\boldsymbol{Y}_{L}=\left[\begin{array}{cc}
Y_{i i}+Y_{i j} & -Y_{i j} \\
-Y_{i j} & Y_{j j}+Y_{i j}
\end{array}\right]
$$

where the diagonal contains the sum of conductivities between the corresponding conductor and all other conductors, including the reference one. The conductivity between the respective two conductors is off-diagonal with a minus sign.

The frequency dependent matrix $\boldsymbol{H}(f)$ of the voltage transfers between the individual conductors can be determined using the load admittance matrix and a matrix $\boldsymbol{M}$, which comprises the matrices $A$, $B, C, D$, and is calculated as the product of the matrices $M_{i}$ of the individual multi-ports. The matrix $\boldsymbol{H}(f)$ can be computed as follows:

$$
\boldsymbol{H}(f)=\left(\boldsymbol{A}+\boldsymbol{B} \boldsymbol{Y}_{L}\right)^{-1} .
$$

Consequently, the power line branch ending with the load $Y_{L}$ (a transformer) could be modeled as a multiport that represents the parallel admittance $\boldsymbol{Y}_{p}$. In order to calculate the $\boldsymbol{Y}_{p}$, it is necessary to compute the matrix $M$ of the line, where the $M$ comprises the matrices $A, B, C, D$ (as outlined in Equations (14) and (15)). The resulting admittance of the whole branch can then be calculated as:

$$
\begin{aligned}
\boldsymbol{Y}_{p} & =\boldsymbol{I}_{i n} \boldsymbol{V}_{\text {in }}^{-1}=\left(\boldsymbol{C} \boldsymbol{V}_{L}+\boldsymbol{D} \boldsymbol{I}_{L}\right)\left(\boldsymbol{A} \boldsymbol{V}_{L}+\boldsymbol{B} \boldsymbol{I}_{L}\right)^{-1} \\
& =\left(\boldsymbol{C}+\boldsymbol{D} \boldsymbol{Y}_{L}\right)\left(\boldsymbol{A}+\boldsymbol{B} \boldsymbol{Y}_{L}\right)^{-1} .
\end{aligned}
$$


The multi-port parallel admittance can be represented by an $\boldsymbol{M}_{i}$ matrix. Since the output and input voltages are equal, the matrix $A$ is an identity matrix, and the matrix $B$ embodies a zero matrix. The matrix $C$ then equals the branch admittance matrix $\boldsymbol{Y}_{p}$, and the matrix $\boldsymbol{D}$ is the identity matrix.

\section{Parameters of the Overhead Lines and Cables}

The procedure characterized above can be employed for the modeling of overhead lines and cables as well; however, it is either necessary to know the matrices $R, L, G, C$ or $Z$ and $Y$.

The internal resistance of the line is frequency dependent, and we define it as the matrix $\boldsymbol{R}_{c}(f)$, whose diagonal contains the internal resistances of the individual conductors, caused primarily by the resistivity of the conductor material, the skin effect, and the temperature of the conductor. The elements of the $\boldsymbol{R}_{c}(f)$ can be expressed as:

$$
r_{c i i}=r_{d} k_{\vartheta} k_{A C} \text {. }
$$

where $r_{d}$ is the resistance of $1 \mathrm{~m}$ of the conductor at $20{ }^{\circ} \mathrm{C}$ without the skin effect, $k_{\vartheta}$ is the temperature coefficient of the conductor's resistance, and $k_{A C}$ is the resistance coefficient resulting from the skin effect.

\subsection{Ground Impedance}

Ground impedance was independently defined by Carson [14] and Pollaczek [15]. To evaluate their impedance formula, we need to solve integral terms whose analytical integrations are impossible [16]. While the given integral could not be resolved analytically, a series of approximations were introduced, and these are compared within [17]; an approximation using a logarithmic function is presented in [18].

A very precise solution for our simulations is achievable via computing the Pollaczek-derived integral numerically by the procedure described in [16], where the authors derive the ground impedance formula:

$$
z_{e}(j \omega)=\frac{j \omega \mu_{0}}{2 \pi} \int_{0}^{\frac{\pi}{2}} \frac{2 e^{-H \tan (\Phi)}}{\tan (\Phi)+\sqrt{\tan ^{2}(\Phi)+j \omega \mu_{e} \sigma_{e}}} \frac{\cos (x \tan (\Phi))}{\cos ^{2}(\Phi)} d \Phi .
$$

where the $H$ represents the relevant height of the overhead conductor or, alternatively, depth of the underground conductor; $x$ represents the mutual conductor horizontal distances; $\mu_{e}$ is the corresponding air/soil permeability; and $\Phi$ is a transformed integration variable. All of the quantities are described in more detail within [16]. The conductivity of the ground $\sigma_{e}$ is available in specialized maps and atlases [19]; alternatively, it can be measured, for example, using the magnetotellurics method [20].

\subsection{Overhead Line Parameters}

The numerous methods for the modeling of an overhead power line are discussed within a large set of papers, including $[17,21,22]$.

To facilitate the related calculations, the ground should invariably constitute the reference conductor, regardless of the fact that either the ground or another phase embody the reference for the communication.

The impedance and admittance of an overhead power line can be expressed as:

$$
\begin{aligned}
& \boldsymbol{Z}=\boldsymbol{R}_{c}(f)+\boldsymbol{Z}_{e}(f)+j \omega \boldsymbol{L}, \\
& \boldsymbol{Y}=j \omega \boldsymbol{C} .
\end{aligned}
$$

where $\boldsymbol{R}_{c}(f)$ is the internal resistance of the individual conductors defined as described above; $\boldsymbol{Z}_{e}(f)$ is the ground impedance characterized in Section 3.1; $L$, defined below, denotes the inductance matrix of 
an overhead power line; and $C$, also defined below, represents the capacitance matrix of the overhead power line.

The inductance matrix elements can be calculated using the equation for the inductance of a wire over a perfectly conducting surface [16]:

$$
l=\frac{\mu_{0}}{2 \pi}\left(\ln \frac{D_{2}}{D_{1}}\right) .
$$

In the case of elements located on the matrix diagonal (denoted $\left.l_{i i}\right), D_{1}$ and $D_{2}$ are:

$$
\begin{aligned}
& D_{1}=r_{i}, \\
& D_{2}=2 h_{i} .
\end{aligned}
$$

where $r_{i}$ is the radius of the relevant conductor, and $h_{i}$ denotes the clearance between the given conductor and the ground.

In elements outside the matrix diagonal (denoted $\left.l_{i j}\right), D_{1}$ and $D_{2}$ are expressed as follows:

$$
\begin{aligned}
& D_{1}=\sqrt{\left(h_{i}-h_{j}\right)^{2}+x_{i j}^{2}}, \\
& D_{2}=\sqrt{\left(h_{i}+h_{j}\right)^{2}+x_{i j}^{2}} .
\end{aligned}
$$

where $h_{i}$ and $h_{j}$ denote the conductor-to-ground distance, and $x_{i j}$ is the horizontal distance between the given conductors.

The capacitance of an overhead power line can be determined using the formula for conductors in a homogeneous environment [21]:

$$
C \boldsymbol{L}=\boldsymbol{L C}=\mu_{0} \varepsilon_{0} \mathbf{1}_{n} \Rightarrow \boldsymbol{C}=\mu_{0} \varepsilon_{0} \boldsymbol{L}^{-1} .
$$

where $\varepsilon_{0}$ is the permittivity and $\mu_{0}$ the permeability of vacuum. How to measure line-to-ground capacitance is described in [23].

\subsection{Underground Power Lines (Cables)}

The shielding is considered the reference conductor. This also applies where shielding is not used as a reference for the communication signal transmission. If an unscreened cable is used, the ground can be regarded as shielding with an infinite radius.

The matrix $\boldsymbol{R}(f)$ constitutes the sum of the above-defined matrices $\boldsymbol{R}_{c}$, which represent the impedances of the individual cable conductors, and $\boldsymbol{R}_{s}$, whose dimension is identical to that of the matrix $\boldsymbol{R}_{c}$; all elements of the matrix $\boldsymbol{R}_{s}$ exhibit a value equal to the internal resistance of the shielding, according to Equation (19). In an unscreened conductor, the matrix $\boldsymbol{Z}_{e}$ is utilized instead of the $\boldsymbol{R}_{s}$, as outlined in Section 3.1, where the conductor-to-ground clearance equals $c_{1}-b_{i}[17,24]$.

The inductance matrix could be defined according to [25] as:

$$
\begin{aligned}
& l_{i i}=\frac{\mu_{i}}{2 \pi} \ln \frac{c_{1}^{2}-b_{i}^{2}}{c_{1} a_{i}}, \\
& l_{i j}=\frac{\mu_{i}}{4 \pi} \ln \frac{c_{1}^{4}-\left(b_{i} b_{j}\right)^{2}-2\left(b_{i} b_{j}\right) c_{1}^{2} \cos \theta_{i j}}{c_{1}^{2}\left(b_{i}^{2}+b_{j}^{2}-2 b_{i} b_{j} \cos \theta_{i j}\right)} .
\end{aligned}
$$

where $\mu_{i}$ is the absolute magnetic permeability of the internal insulation. The meaning of the other variables is shown in Figure 2. 


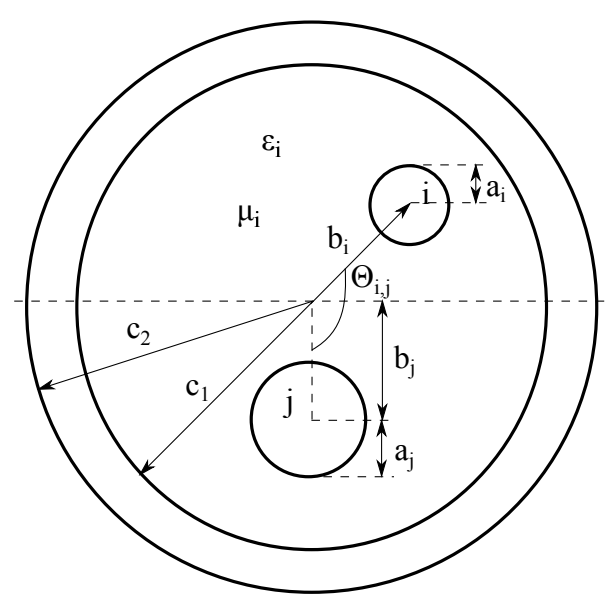

Figure 2. A diagram of a shielded cable [25].

The cable capacitance can be determined from the formula [21] as:

$$
C L=L C=\mu_{i} \varepsilon_{i} \mathbf{1}_{n} \Rightarrow C=\mu_{i} \varepsilon_{i} L^{-1} .
$$

where $\varepsilon_{i}$ is the permittivity of the internal insulation, and $\mu_{i}$ denotes the absolute magnetic permeability of the internal insulation.

The transverse conductivity of the cable is definable, according to the formula proposed in [26], as:

$$
\frac{G}{C}=\frac{\sigma_{i}}{\varepsilon_{i}} \Rightarrow G=\frac{C \sigma_{i}}{\varepsilon_{i}} .
$$

where $\sigma_{i}$ is the conductivity of the internal insulation, and $\varepsilon_{i}$ denotes the permittivity of the internal insulation.

\section{Transformer Model}

Several research reports, for example [27], present transformer models, but they are not suitable for a multi-line PLC model. Since the communication in typical PLC systems is not intended to pass through a transformer, our transformer is modelled just as a load. Such an architecture corresponds to the requirements of the typical MV/LV grids operated in the European Union. A model of a real transformer is shown in Figure 3. The wires a, b, c, n are introduced only to connect the load representing a low voltage network without a transformer. The model does not allow us to describe the transmission through the transformer but is suitable for describing the transformer as a multi-line load. The parameters for the given model were obtained via measurements on a transformer manufactured by BEZ BRATISLAVA, type T0326/22 10000/400(231) V 200 kVA (hereafter, we use the abbreviated code $\mathrm{T} 0326 / 22)$.

If the transformer is symmetrical and undamaged, the values of the model elements indicated in Figure 3 are identical for all the phases. We then have:

$$
\begin{aligned}
& R_{1}=R 4=R 5=R 6 \\
& L_{1}=L 4=L 5=L 6 \\
& C=C 2=C 3=C 4 \\
& R_{2}=R 7=R 8=R 9 \\
& L_{2}=L 1=L 2=L 3 \\
& R_{3}=R 1=R 2=R 3
\end{aligned}
$$

In Figure 3, the points $A, B$, and $C$ represent the phases on the medium-voltage side; $G$ denotes the ground on the medium-voltage side; $a, b, c$ are the phases on the low-voltage side; and $n$ is a neutral 
conductor or the ground on the low-voltage side. The load of the low voltage side is represented by $Z_{L V}$ and is connected to the points $a n, b n$, and $c n$. It is assumed that the load is symmetrical; therefore, each node is loaded with the same impedance $Z_{L V}$. To simplify the notation, the impedances $Z_{1}, Z_{2}, Z_{3}$ can be introduced:

$$
\begin{aligned}
& Z_{1}=\frac{i \omega R_{1} L_{1}}{R_{1}+i \omega L_{1}} \\
& Z_{2}=\frac{i \omega R_{2} L_{2}}{R_{2}+i \omega L_{2}}+R_{3} \\
& Z_{3}=\frac{1}{i \omega C} .
\end{aligned}
$$

The impedance between a phase and the ground is:

$$
Z_{L G}=\frac{3 Z_{1}\left(Z_{2}+Z_{L V}\right) Z_{3}+Z_{1} Z_{3}^{2}+\left(Z_{2}+Z_{L V}\right) Z_{3}^{2}}{3 Z_{1}\left(Z_{2}+Z_{L V}\right)+3 Z_{1} Z_{3}+3\left(Z_{2}+Z_{L V}\right) Z_{3}}
$$

and the impedance between the phases is:

$$
Z_{L L}=\frac{2 Z_{1}\left(Z_{2}+Z_{L V}\right) Z_{3}}{Z_{1} Z_{3}+\left(Z_{2}+Z_{L V}\right) Z_{3}+Z_{1}\left(Z_{2}+Z_{L V}\right)}
$$

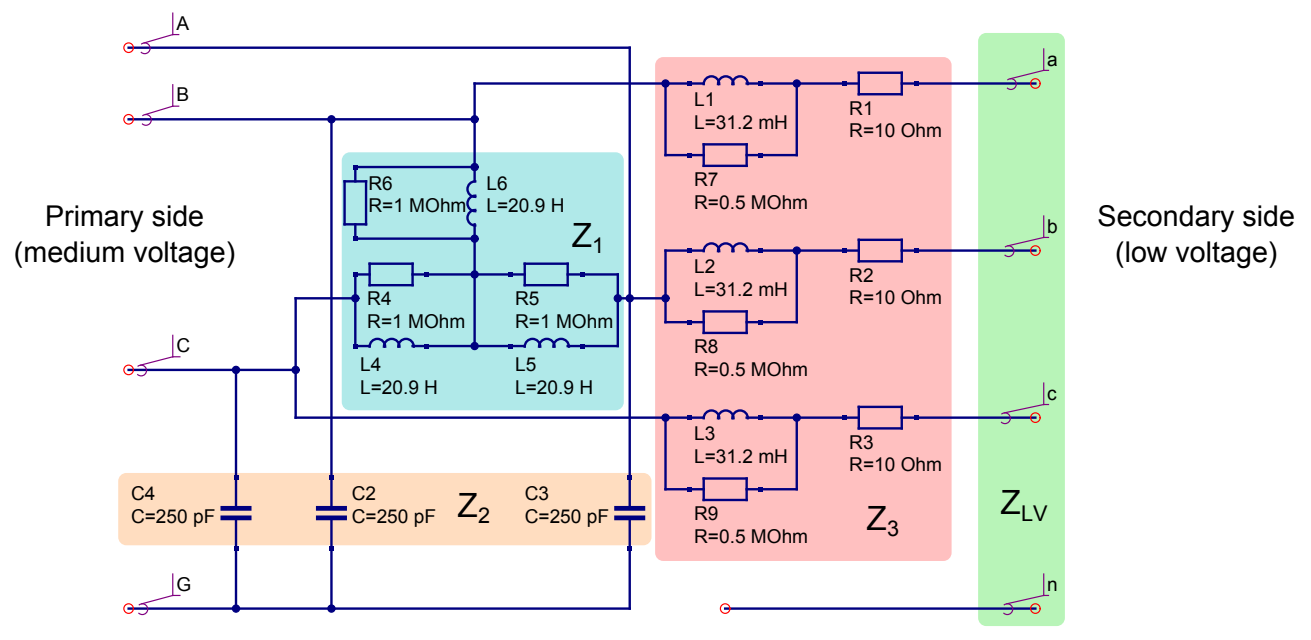

Figure 3. A transformer impedance model to simulate the power line communication (PLC) communication in a medium-voltage line.

A good match between the designed model and the real transformer is illustrated in Figures 4 and 5, which compare the measured and modeled impedances of a transformer with a short-circuit on the low-voltage side. Importantly, the model exhibits satisfactory agreement also with open-circuit measurements on the low-voltage side.

For the frequency bands that are relevant for the PLC communication, the typical impedance of the medium-voltage side of a transformer ranges from hundreds of $\Omega$ to tens of $\mathrm{k} \Omega$; however, for frequencies in close proximity to the resonant frequencies, the impedance may rise up to thousands of $\mathrm{k} \Omega$. It can be observed that the impedance between a phase and the ground is lower than that between the phases. The model aims to address frequencies below $1 \mathrm{MHz}$, as such solution facilitates the modeling of virtually all narrowband power line communications.

The measurements described in [27], and Ref. [28] enable us to claim that the model is suitable for simulating most MV/LV transformers, albeit with the necessity to adjust the resistance, inductance, and capacitance by using the resonant frequencies of the relevant transformer. In individual transformers, 
the resonant frequencies are often specified within the data sheet, as such information is used for Sweep Frequency Response Analysis tests.

Considering the measurements presented in $[29,30]$, the model can be further characterized as applicable for hight-voltage (HV)/MV transformers too; our own measurements and the results discussed within [27] nevertheless also show that the model is unfortunately not suitable for simulating the $\mathrm{LV}$ side of an MV/LV transformer.
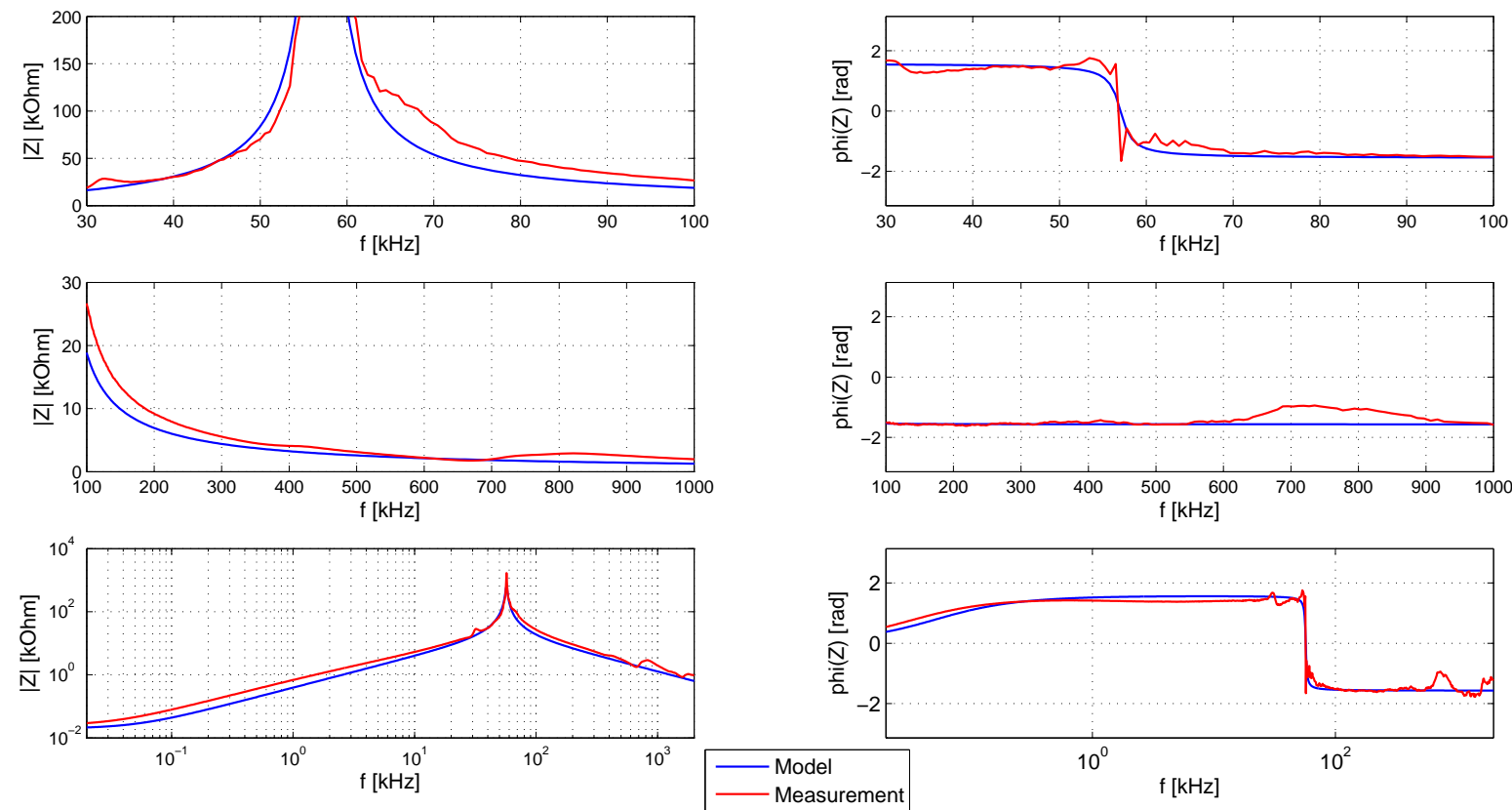

Figure 4. A comparison of the measured and modeled impedances of the T0326/22 transformer in-between the phases of the medium voltage side, with a short circuit at the low voltage side.
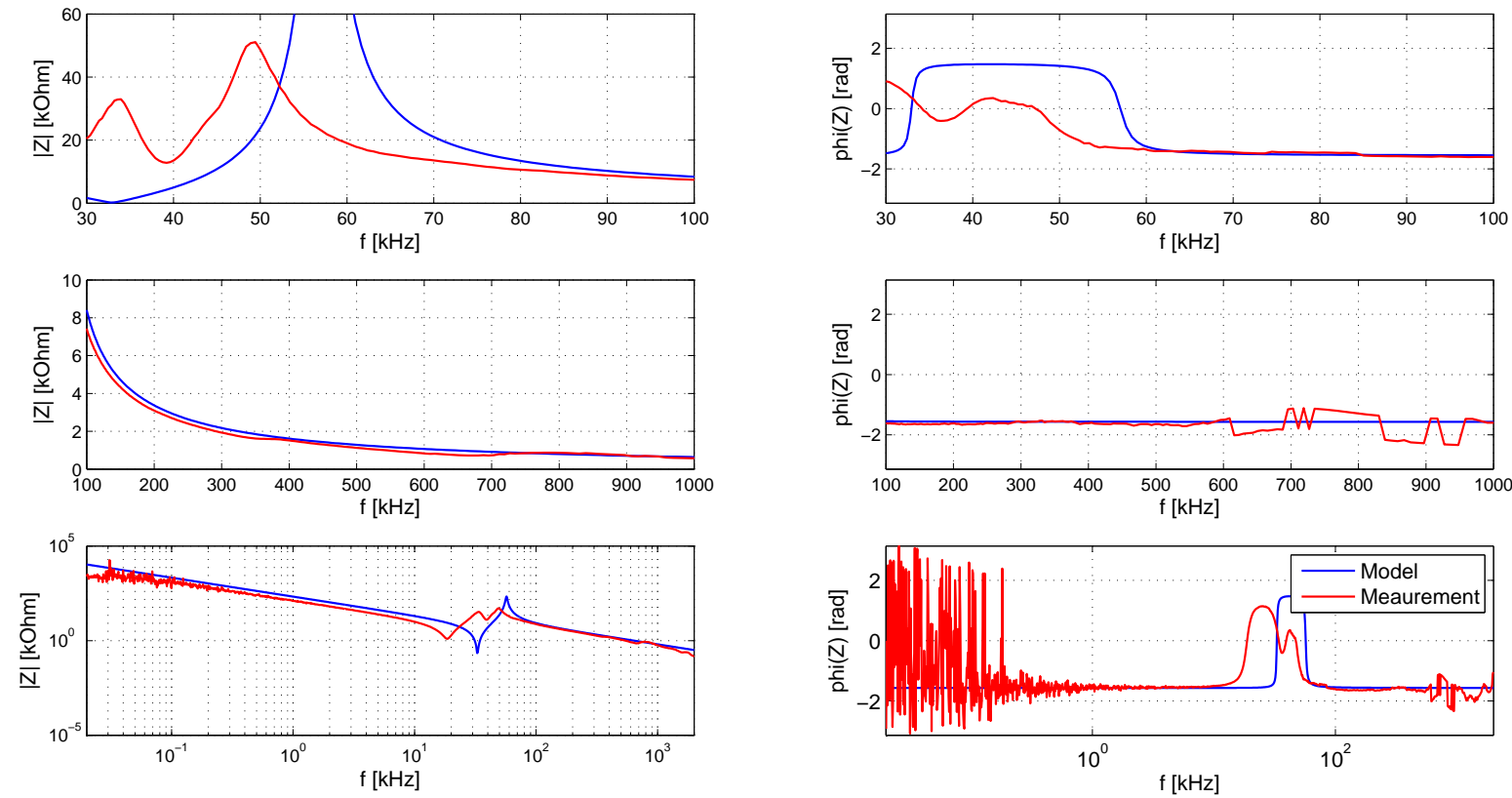

Figure 5. A comparison of the measured and modeled impedances of the T0326/22 in-between the phases and the ground of the medium voltage side, with a short circuit at the low-voltage side. 


\subsection{Signal Transmission through the Transformer}

In our experiments, we used MV/LV a T0326/22 transformer (BEZ Bratislava, Bratislava, Slovakia). The measured voltage transmission through the T0326/22 transformer is represented in Figures 6 and 7. The transmissions BA->an and an->BA were not measured. The transmission of a high frequency PLC signal over a transformer was not relevant for the modelling, as PLC communication within medium voltage lines is not intended to pass to a low voltage network. However, even though the transmission was not modelled, relevant measurement may reveal if the signal can pass from one network to another, causing noise in the latter. The voltage transmission from the medium-voltage side to the low-voltage one for the frequency range of between $30 \mathrm{kHz}$ and $100 \mathrm{kHz}$ is approximately $-33 \mathrm{~dB}$, and we assume that the attenuation depends also on the $50 \mathrm{~Hz}$ voltage ratio of the transformer. For the frequencies from $100 \mathrm{kHz}$ to $1 \mathrm{MHz}$, the transmission varies between $-33 \mathrm{~dB}$ and $-55 \mathrm{~dB}$. As the measurement was performed at the impedance of $50 \Omega$, and the typical low-voltage network impedance is significantly lower, the real attenuation of the signal could be even higher. The voltage transmission from the low-voltage side to the medium-voltage one for the frequencies between $30 \mathrm{kHz}$ and $100 \mathrm{kHz}$ decreases with the frequency, from $-17 \mathrm{~dB}$ to $-27 \mathrm{~dB}$. At the frequencies from $100 \mathrm{kHz}$ to $1 \mathrm{MHz}$, the transmission varies again, within the range of $-25 \mathrm{~dB}$ to $-45 \mathrm{~dB}$. Due to the comparatively high impedance of medium-voltage networks at the assumed frequencies, the real attenuation is expected to be lower.

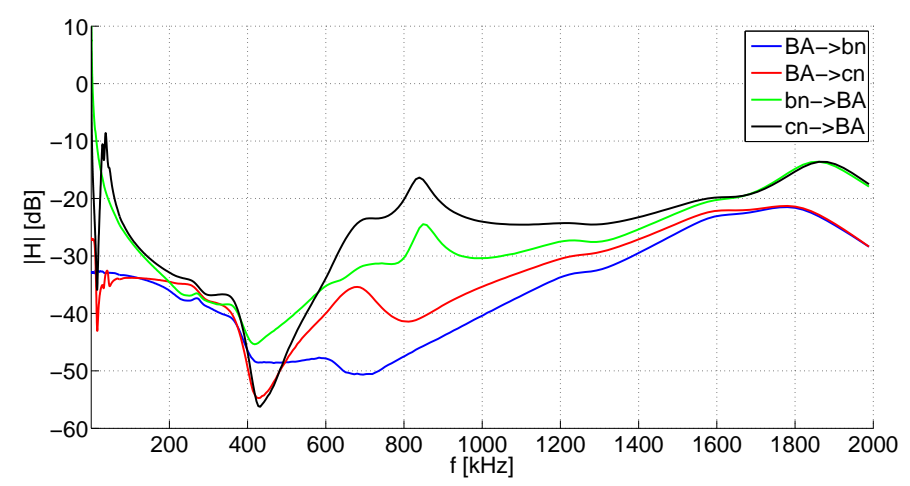

Figure 6. The measured voltage transmission through the T0326/22 transformer, $20 \mathrm{~Hz}$ to $2 \mathrm{MHz}$.

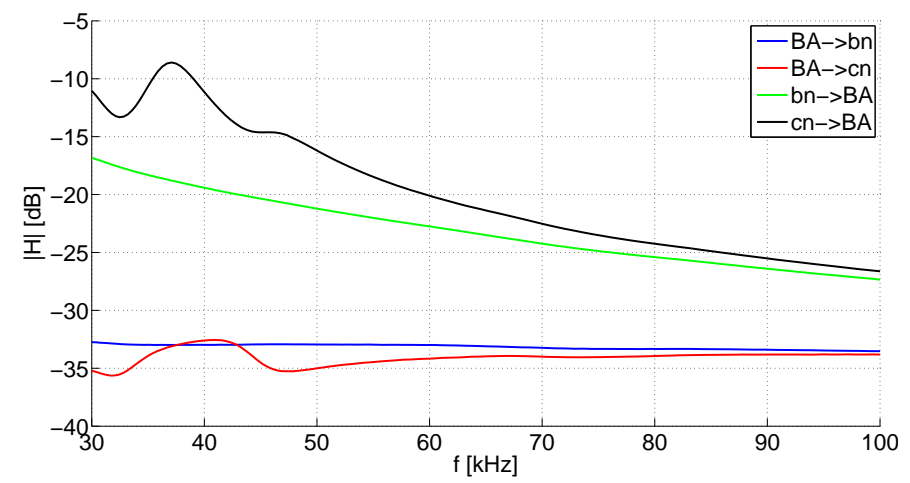

Figure 7. The measured voltage transmission through the T0326/22 transformer, $30 \mathrm{kHz}$ to $100 \mathrm{kHz}$.

Based on the attenuation values described above, it is possible to assume that the signal transmission from an MV network to an LV one constitutes a critical aspect for any bidirectional communication across a transformer. With respect to the fact that, in LV networks, the communication device is most often located in the immediate vicinity of the transformer, the success rate of the actual communication depends predominantly on the distance to the other point upon the medium-voltage 
line. For inter-network communication purposes, it is preferable to use frequencies up to $100 \mathrm{kHz}$. If two independent networks operate at the same frequency, interferences will occur, especially on the MV side.

\section{Noise Model}

Using the power spectral density (PSD) of the transmitted signal, the transfer function of channel, and the PSD of the noise at the receiver, the signal-to-noise ratio (SNR) can be established to determine the packet loss rate in a concrete type of communication.

On medium-voltage lines, three types of noise [31] are commonly found-background noise, periodic impulse noise and the non-periodic impulse noise.

The first of these is background noise, whose magnitude decreases with the increasing frequency. The measurement of noise in medium-voltage lines, however, is not discussed in the literature as often as noise measurement in low-voltage lines; therefore, the noise was measured in the medium-voltage network operated by Trinec ironworks (the largest manufacturer of steel in the Czech Republic). The measurements were performed at two locations, with 1142 measuring cycles in total. The average noise was obtained via averaging, from all the measurements, the power spectral density values for the individual frequencies. The maximum and minimum noises constitute the maxima or minima of the power spectral densities for the given frequencies. The example PSDs of the noise are shown in Figure 8 . The results can be compared to the data acquired within similar research carried out around Shizuishan City, China [32]. In the frequency range of up to $1 \mathrm{MHz}$, the noise measured at the two points in Trinec remained between the values from the Hebin and Taole areas, Shizuishan City. In this context, it can be then assumed that the noise values of -60 to $-80 \mathrm{~dB}(\mathrm{~mW} / \mathrm{Hz})$ are common in medium-voltage lines.

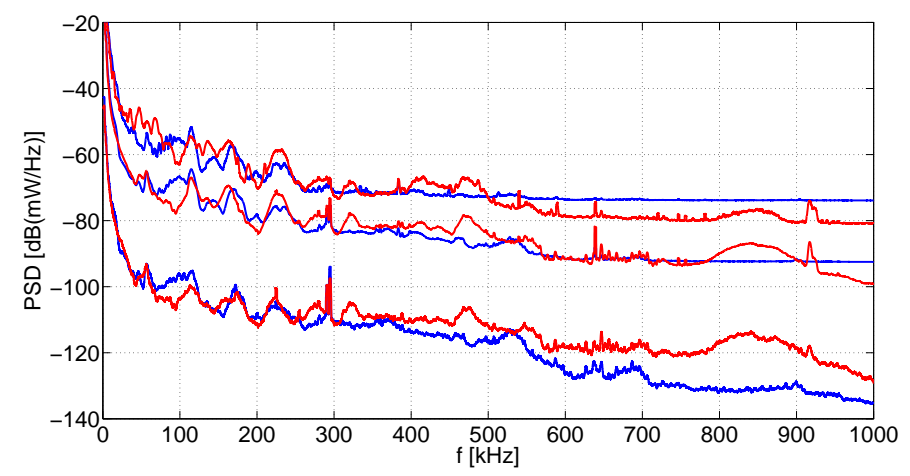

Figure 8. The measured minimum, medium, and maximum power spectral densities of noise in the area of Trinec ironworks, blue-Station 1, red-Station 2.

Periodic synchronous and asynchronous impulse noises were observed on a number of measurements, for example, the cycle shown in Figure 9. However, not all of the samples exhibited significant periodic synchronous interference.

At some moments, impulse asynchronous noise could be measured in the investigated area of the ironworks; this was caused by the startup or shutdown of a technological process. 


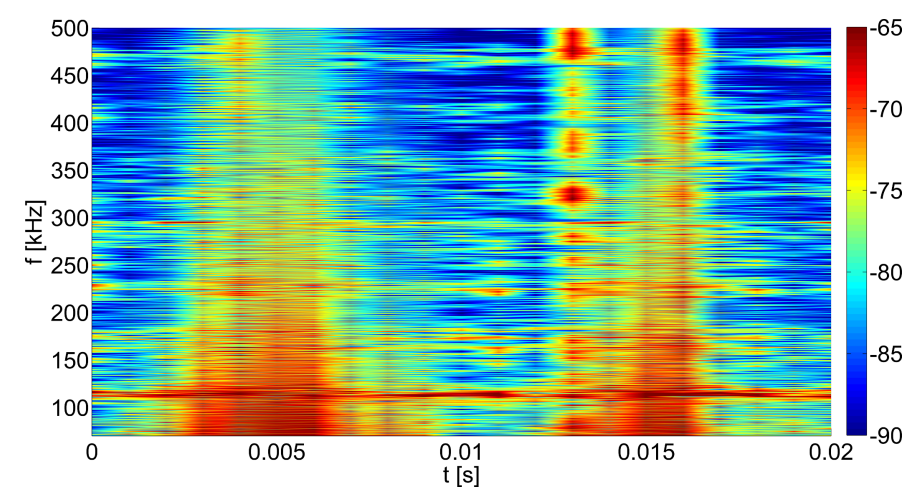

Figure 9. The measured power spectral density of the noise $[\mathrm{dB}(\mathrm{mW} / \mathrm{Hz})]$ in the medium voltage lines at station 2 (Trinec ironworks) during one period of a $50 \mathrm{~Hz}$ signal.

\section{Model of Power Line Communication in Trinec Ironworks}

Based on Sections 2-4.1, it was possible to create models intended for simulating the real communication paths of Trinec ironworks $22 \mathrm{kV}$ power lines.

The modeled topology (Figure 10) comprises an active HV/MV transformer at the distance of $d_{H V}=10 \mathrm{~m}$ from the crossing point; an $\mathrm{MV} / \mathrm{MV}$ arc furnace transformer at the distance of $d_{\text {furnance }}=2 \mathrm{~m}$ from the crossing point; and an MV/LV transformer, whose location from the crossing point is defined as $d_{L V}=2 \mathrm{~km}$. At a distance $x$ (not known exactly but estimated to be $2 \mathrm{~km}$ ), a backup $\mathrm{HV} / \mathrm{MV}$ transformer is placed, and the line is interrupted immediately before it. The modems are located at the crossing point and at the end of the line having the length $x$ that leads towards the backup HV/MV transformer; here, the modems are found right before the disconnection. The three phase line consists of 12 AYKCY $1 \times 240 / 25$ cables, four per each phase.

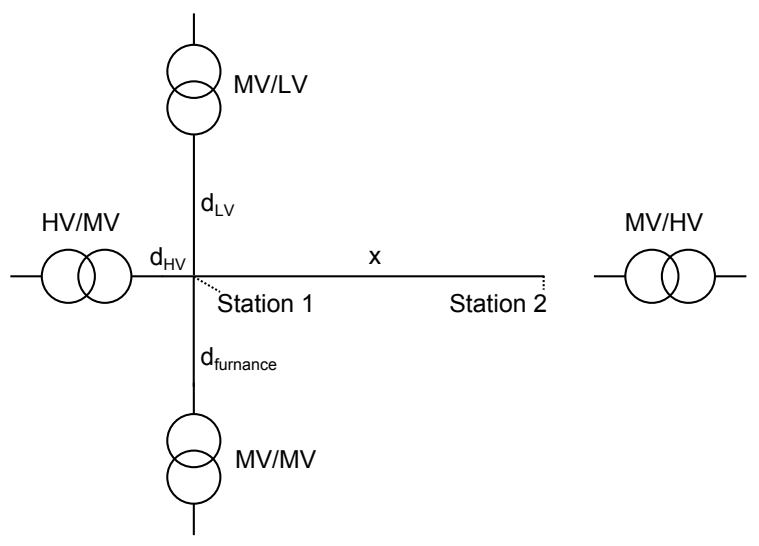

Figure 10. The topology of the medium-voltage distribution network in the Trinec ironworks industrial park.

Considering successful communication, the maximum possible attenuation depends on a broad set of aspects, including the range of frequencies to facilitate the actual communication process, the crest factor, the number of phases into which the signal is transmitted, the bit error ratio (BER)/SNR characteristic, and the current noise level. The most common threshold for satisfactory communication (the 133B frame is received with a $50 \%$ success) consists in the attenuation of the transmission channel between $-38 \mathrm{~dB}$ and $-76 \mathrm{~dB}$; the related simulations are shown in Figures 11 and 12 below. As the discussed line is of the MV type and does not comprise many branches, the simulation shows that it could be possible to communicate over distances of up to tens of kilometers. 


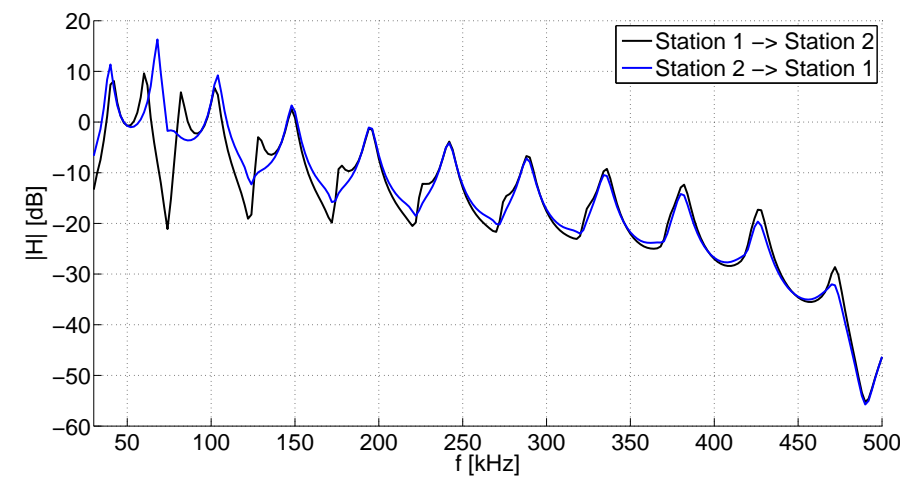

Figure 11. The simulated voltage transmission within Trinec ironworks in relation to the frequency (for the distance of $x=2000 \mathrm{~m}$ ).

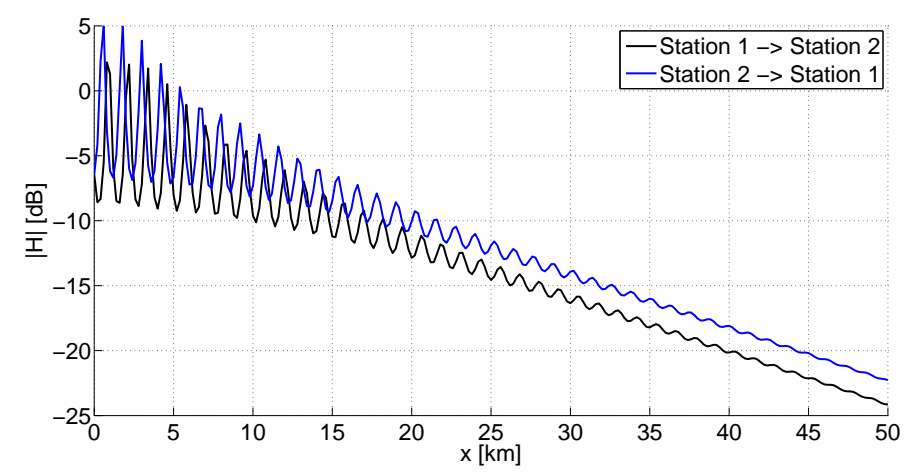

Figure 12. The simulated voltage transmission within Trinec ironworks in relation to the distance (for the frequency of $f=80 \mathrm{kHz}$ ).

The real cable length equaled to approximately $2 \mathrm{~km}$, and thus the measured attenuation of the transmission channel in Figure 13 for the frequencies from $60 \mathrm{kHz}$ to $100 \mathrm{kHz}$ is insignificant and usually does not drop below $-10 \mathrm{~dB}$. These observations sufficiently correspond to the simulated transmission at the given frequencies, as shown in Figure 11. In both the measured and the simulated transmission, it is also possible to observe a certain ripple in the frequency domain.

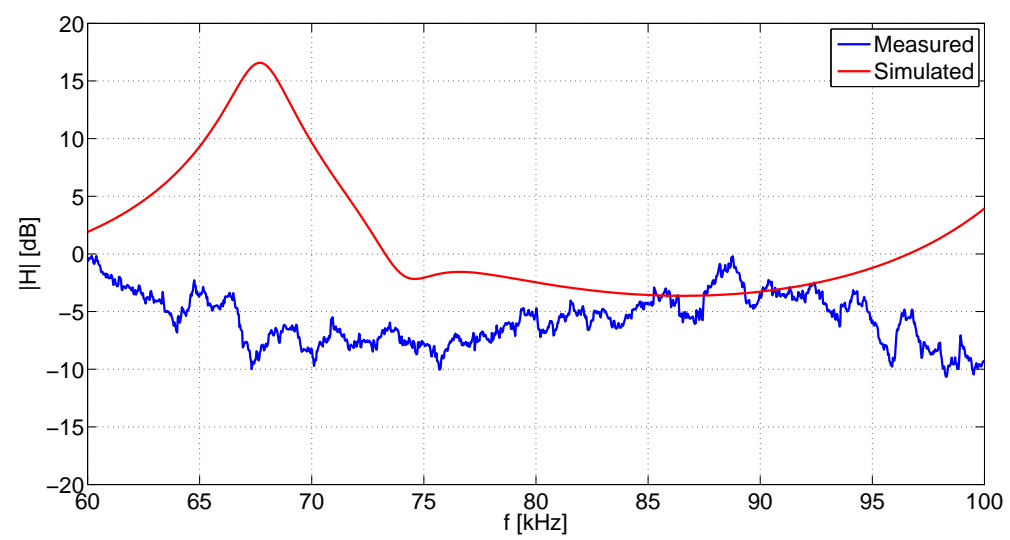

Figure 13. The measured and simulated transmission within Trinec ironworks in relation to the frequency (for the approximate distance of $x \cong 2 \mathrm{~km}$ ).

\section{Conclusions}

At present, multiconductor medium voltage powerline models are used only rarely. We proposed in this paper a multiconductor model suitable for the modelling of overhead multi-phase lines as 
well as multi-conductor cables. To validate the model, we examined a medium-voltage network at a steelworks company, and our simulation and measurement confirmed the ability to communicate at the given distance. The difference between the measured and the simulated transmissions was from $0 \mathrm{~dB}$ to $26 \mathrm{~dB}$ for the individual frequencies. The differences are due to the fact that, at the measured distance of $2 \mathrm{~km}$, the wires had very low attenuation, and the influence of the unmodelled network elements was more pronounced. At longer distances, it can be assumed that the match between simulation and measurement will improve.

The model was developed based on a mathematical representation of the multiconductor lines and transformers found within the research area. The simulation model enables us to not only simulate the PLC capability of the multi-conductor line at a high degree of accuracy but also to evaluate a large number of related aspects, including the following issues:

- $\quad$ the selection of the reference conductor (phase or ground);

- the ability to receive a signal from a phase other than the one into which the signal is transmitted;

- the ability to use different phases of the line as independent communication channels;

- the signal transmission from one network to another via cables laid in parallel.

The above factors cannot be simulated in two-wire models. Importantly, the overall model enables us to estimate the maximum communication distance in the medium voltage network, and it can be utilized to assess the impact of changes in the communication system; such changes comprise the range of applied frequencies, the crest factor, the BER/SNR characteristics, the compensation of the coupling attenuation, and other alterations to improve the communication speed and maximum distance.

Based on the MV side of the transformer model and considering its comparison with measurements presented in Figures 4 and 5, it is possible to argue that the impedance at PLC frequencies in MV grids is significantly higher than in LV ones. Furthermore, the average noise measured on $22 \mathrm{kV}$ lines at Trinec ironworks (Figure 8) varied for the relevant frequencies from $-100 \mathrm{~dB}$ to $-60 \mathrm{~dB}$ and is comparable with the measurements in ShiZuishan City, China [32]. These facts facilitate the operation of the PLC in the underlying medium-voltage grid, where greater communication distances are required.

The multiconductor model described herein could be extended with a sensitivity analysis, allowing us to quantify the influence of each parameter on the communication success ratio. The key model parameters that affect the communication success ratio include-for example, the resistance, capacitance, and inductance of the conductors, the transformer impedance, the line length, and the location of the branches. The relevant sensitivity analysis could specify the parameters to be known exactly as well as those in which only an approximate is sufficient; such extension of the model, however, reaches beyond the scope of this paper.

Acknowledgments: The research activities were supported by Grant No. FEKT-S-17-4234-“'Industry 4.0 in Automation and Cybernetics" - the Internal Science Fund of Brno University of Technology, Czech Republic.

Author Contributions: Lesek Franek is the main author of the article, who also developed the models and performed the necessary experiments. Petr Fiedler, the co-author, provided his theoretical knowledge of modeling, cooperated on developing the model, and supervised the research activities.

Conflicts of Interest: The authors declare no conflict of interest.

\section{References}

1. Schwartz, M. Carrier-wave telephony over power lines: Early history [History of Communications]. IEEE Commun. Mag. 2009, 47, 14-18.

2. Broadbridge, R. Power line modems and networks. In Proceedings of the Second IEE National Conference on Telecommunications, York, UK, 2-5 April 1989; pp. 294-296.

3. Sendin, A.; Pena, I.; Angueira, P. Strategies for Power Line Communications Smart Metering Network Deployment. Energies 2014, 7, 2377-2420. 
4. Nassar, M.; Lin, J.; Mortazavi, Y.; Dabak, A.; Kim, I.H.; Evans, B. Local Utility Power Line Communications in the 3-500 kHz Band: Channel Impairments, Noise, and Standards. IEEE Signal Process. Mag. 2012, 29, 116-127.

5. Liu, Z.; Ma, X.; Yang, J.; Li, J. Influence of single-phase short circuit to TWACS signal. In Proceedings of the 2013 International Conference on Quality, Reliability, Risk, Maintenance, and Safety Engineering (QR2MSE), Chengdu, China, 15-18 July 2013; pp. 1800-1803.

6. Mak, S.T. A New Method of Generating TWACS Type Outbound Signals for Communication on Power Distribution Networks. IEEE Trans. Power Appar. Syst. 1984, PAS-103, 2134-2140.

7. Galli, S.; Scaglione, A.; Wang, Z. For the Grid and Through the Grid: The Role of Power Line Communications in the Smart Grid. Proc. IEEE 2011, 99, 998-1027.

8. Atayero, A.A.; Alatishe, A.; Ivanov, Y.A. Power Line Communication Technologies: Modeling and Simulation of PRIME Physical Layer. World Congr. Eng. Comput. Sci. 2012, 2, 931-936.

9. Llano, A.; Angulo, I.; Angueira, P.; Arzuaga, T.; de la Vega, D. Analysis of the Channel Influence to Power Line Communications Based on ITU-T G.9904 (PRIME). Energies 2016, 9, 39.

10. Ikpehai, A.; Adebisi, B.; Rabie, K. Broadband PLC for Clustered Advanced Metering Infrastructure (AMI) Architecture. Energies 2016, 9, 569.

11. Zhang, H.T.; Lai, L. An overview on smart grid simulator. In Proceedings of the 2012 IEEE Power and Energy Society General Meeting, San Diego, CA, USA, 22-26 July 2012; pp. 1-6.

12. Antonini, G.; Orlandi, A.; Pignari, S. Review of Clayton R. Paul Studies on Multiconductor Transmission Lines. IEEE Trans. Electromagn. Compat. 2013, 55, 639-647.

13. Versolatto, F.; Tonello, A. An MTL Theory Approach for the Simulation of MIMO Power-Line Communication Channels. IEEE Trans. Power Deliv. 2011, 26, 1710-1717.

14. Carson, J.R. Wave propagation in overhead wires with ground return. Bell Syst. Tech. J. 1926, 5, 539-554.

15. Pollaczek, F. Uber das Feld einer unendlich langen wechselstromdurchflossenen Einfachleitung. ENT Band 3 1926, 33, 339-360. (In Japanese)

16. Noda, T. Numerical techniques for accurate evaluation of overhead line and underground cable constants. IEEJ Trans. Electr. Electr. Eng. 2008, 3, 549-559.

17. Uribe, F. Assessing closed-form approximations for underground cable earth impedances. In Proceedings of the 2003 IEEE Power Engineering Society General Meeting, Toronto, ON, Canada, 13-17 July 2003; pp. 649-654.

18. Anatory, J.; Theethayi, N. On the Efficacy of Using Ground Return in the Broadband Power-Line Communications-A Transmission-Line Analysis. IEEE Trans. Power Deliv. 2008, 23, 132-139.

19. International Telecommunication Union. World Atlas of Ground Conductivities; Technical Report P.832-3; International Telecommunication Union: Geneva, Switzerland, 2012.

20. Naidu, G.D. Magnetotellurics: Basic Theoretical Concepts. In Deep Crustal Structure of the Son-Narmada-Tapti Lineament, Central India; Springer: Berlin/Heidelberg, Germany, 2012; pp. 13-35.

21. Paul, C.R. Solution of the transmission-line equations for lossy conductors and imperfect earth. Proc. Inst. Electr. Eng. 1975, 122, 177-182.

22. Kim, J.H.; Kim, J.Y.; Cho, J.T.; Song, I.K.; Kweon, B.M.; Chung, I.Y.; Choi, J.H. Comparison between Underground Cable and Overhead Line for a Low-Voltage Direct Current Distribution Network Serving Communication Repeater. Energies 2014, 7, 1656-1672.

23. Yang, Q.; Zhang, B.; Ran, J.; Chen, S.; He, Y.; Sun, J. Measurement of Line-to-Ground Capacitance in Distribution Network Considering Magnetizing Impedance's Frequency Characteristic. Energies 2017, $10,477$.

24. Theethayi, N.; Thottappillil, R.; Paolone, M.; Nucci, C.A.; Rachidi, F. External impedance and admittance of buried horizontal wires for transient studies using transmission line analysis. IEEE Trans. Dielectr. Electr. Insul. 2007, 14, 751-761.

25. Kane, M.; Ahmad, A.; Auriol, P. Multiwire shielded cable parameter computation. IEEE Trans. Magn. 1995, 31, 1646-1649.

26. Farahmand, F. Introduction to Transmission Lines. In A Primer on Electromagnetic Fields; International Publishers Association: Geneva, Switzerland, 2012. 
27. Kikkert, C. A PLC frequency model of 3 phase power distribution transformers. In Proceedings of the 2012 IEEE Third International Conference on Smart Grid Communications (SmartGridComm), Tainan, Taiwan, 5-8 November 2012; pp. 205-210.

28. Sriphuek, R.; Chotigo, S. Low-cost frequency response analyzer for transformer diagnosis. In Proceedings of the 2012 International Conference on Condition Monitoring and Diagnosis (CMD), Bali, Indonesia, 23-27 September 2012; pp. 437-441.

29. Pham, D.; Pham, T.; Borsi, H.; Gockenbach, E. A new method for purposes of failure diagnostics and FRA interpretation applicable to power transformers. IEEE Trans. Dielectr. Electr. Insul. 2013, 20, 2026-2034.

30. Brandt, M.; Peniak, A. Identification of the power transformer 110/23 kV failure. In Proceedings of the 2014 ELEKTRO, Rajecke Teplice, Slovakia, 19-20 May 2014; pp. 531-534.

31. Zimmermann, M.; Dostert, K. An analysis of the broadband noise scenario in powerline networks. In Proceedings of the International Symposium on Powerline Communications and its Applications (ISPLC2000), Limerick, Ireland, 5-7 April 2000; pp. 5-7.

32. Zheng, T.; Yang, X.; Zhang, B.; Chen, J.; Y, Z.; Tian, T. Research of Noise Characteristics for 10-kV Medium-Voltage Power Lines. IEEE Trans. Power Deliv. 2007, 22, 142-150.

(C) 2017 by the authors. Licensee MDPI, Basel, Switzerland. This article is an open access article distributed under the terms and conditions of the Creative Commons Attribution (CC BY) license (http:/ / creativecommons.org/licenses/by/4.0/). 